\title{
Toward a new understanding of disk galaxy formation
}

\author{
Susan Kassin \\ Space Telescope Science Institute, USA
}

\begin{abstract}
One of the most important open issues in astronomy is the assembly of galactic disks. Over the last decade this has been addressed with large surveys of the internal kinematics of galaxies spanning the last 10 billion years of the universe. I will discuss recent results from the field that show the kinematic assembly of disk galaxies since a redshift of 2.5 , including recent deep 10-30 hour observations by my group with the DEIMOS spectrograph on Keck. These results strongly challenge traditional analytic models of galaxy formation and provide an important benchmark for simulations. Furthermore, I will discuss our plans for extending measurements to higher redshifts with future instruments such as the JWST's NIRSpec IFU and the E-ELT's MOSAIC and HARMONI IFUs. From mock JWST and E-ELT observations of simulated galaxies, we are learning that interpreting these observations of galaxies in the early universe, when merging is frequent, is not necessarily straightforward.
\end{abstract}

